\title{
Reversal of P-glycoprotein-mediated multidrug resistance in human sarcoma MES-SA/Dx-5 cells by nonsteroidal anti-inflammatory drugs
}

\author{
ANTONIO ANGELINI, MANUELA IEZZI, CONCETTA DI FEBBO, \\ CARMINE DI ILIO, FRANCO CUCCURULLO and ETTORE PORRECA \\ Department of Medicine and Aging, School of Medicine and Aging Research Center, \\ Ce.S.I. 'Gabriele D'Annunzio' University Foundation, Chieti-Pescara, Italy
}

Received April 18, 2008; Accepted July 2, 2008

DOI: $10.3892 /$ or_00000067

\begin{abstract}
Multidrug resistance (MDR) mediated by $\mathrm{P}$ glycoprotein $(\mathrm{P}-\mathrm{gp})$ is one of the major reasons for the failure of cancer therapy. Several chemosensitizers are able to reverse in vitro MDR by inhibiting P-gp, although high toxicity limits their clinical application. In this study, we aimed to investigate the in vitro effectiveness of four common nonsteroidal anti-inflammatory drugs (NSAIDs) such as Curcumin (Cur), Sulindac (Sul), Ibuprofen (Ibu) and NS-398 (NS) to inhibit P-gp activity at clinically achievable doses and to evaluate their potential use as sensitizers in anti-cancer chemotherapy. The human doxorubicin (doxo) resistant uterine sarcoma cells (MES-SA/Dx-5) expressing high levels of P-gp, were treated with different doxo concentrations in the presence or absence of NSAIDs. Cellular accumulation of doxo, cytotoxicity and apoptosis induction were measured in comparison with Verapamil, a specific P-gp inhibitor, used as a reference molecule. We found that Ibu, Cur and NS-398 enhanced significantly doxo retention, cytotoxicity and apoptosis on resistant MES-SA/Doxo-5 cells when compared with doxo alone. In contrast, no significant changes were found in resistant cells treated with Sul-doxo combinations. Our results demonstrate that Ibu, Cur and NS-398 below their therapeutic plasma concentrations were able to overcome P-gp-mediated MDR in MES-SA/Dx-5 cells. These findings provide the rationale for clinical studies of NSAIDs and/or derivatives as a new potential generation of chemosensitizers to improve effectiveness of the anti-cancer drugs in the treatment of human cancer.
\end{abstract}

Correspondence to: Dr Antonio Angelini, Università 'Gabriele D'Annunzio', Facoltà di Medicina e Chirurgia, Centro di Scienze dell'Invecchiamento (Ce.S.I.), Campus Universitario, Via Colle dell'Ara, 66013 Chieti Scalo, Italy

E-mail: aangelini@unich.it

Key words: P-glycoprotein, non-steroidal anti-inflammatory drugs, multidrug resistance, Ibuprofen, Curcumin, Sulindac, NS-398, Verapamil

\section{Introduction}

Multidrug resistance (MDR) of cancer cells constitutes one of the major obstacles to successful treatment of cancer (1-6). MDR is a phenomenon in which tumor cells are resistant to the cytotoxic effects of various structurally and functionally unrelated chemotherapeutic agents (7). The predominant cause of MDR is the overexpression of a $170-\mathrm{kDa}$ plasma membrane P-glycoprotein (P-gp), the product of the multidrug resistance (MDR) gene, an ATP-dependent transporter which acts as an efflux pump decreasing drug accumulation and cytotoxicity (8-11). Previously, several P-gp inhibitors or chemosensitizing agents, have been examined in vitro in combination with cytostatic agents to inhibit the activity of this drug transporter in an effort to improve the effectiveness of anti-cancer therapy. However, the application of these chemical agents in chemotherapy is limited because of their severe toxic side effects (12-19). Recent evidence indicates that non-steroidal anti-inflammatory drugs (NSAIDs) possess an anti-tumor activity in different types of human cancer cell lines (20-25). However, the high concentrations of NSAIDs needed to inhibit cancer cell proliferation, limit their clinical use in cancer chemotherapy. In addition, these compounds exert different biological effects and act on different proteins and could interfere with the processes involved in the cytotoxic action of anti-tumor agents. The aim of this study was to investigate whether NSAIDs at clinically achievable doses, interact with the P-gp membrane transporter. Altered P-gp activity, which may result from these interactions, could modify the absorbability and toxicity of anti-cancer drugs and consequently to influence the clinical response. Thus, NSAIDs could be used in combination with anti-neoplastic agents as chemosensitizers to inhibit the transport processes P-gpmediated and to improve the treatment responsiveness in cancer patients. In the present study, we analyzed the in vitro effects of four common NSAIDs, Ibuprofen (Ibu), Curcumin (Cur), NS-398 (NS) and Sulindac (Sul) on MDR of MESSA/Dx-5 human sarcoma cell line, which express high levels of P-gp $(26,27)$. The doxo-resistant sarcoma cells were treated with two different doxo concentrations in the presence or absence of a concentration of NSAIDs attainable in human serum and intracellular doxo accumulation, doxo 
cytotoxicity and apoptosis induction was determined. We have used doxo concentrations which can be achieved in the blood of patients who undergo anti-cancer treatment. As a positive control, we used verapamil (Ver), a specific inhibitor for P-gp that blocks binding of drugs to P-gp competing against anti-cancer drug for binding site on P-gp (28).

\section{Materials and methods}

Chemicals and drugs. Doxorubicin (doxorubicin hydrochloride), was obtained from ICN Biomedicals Inc. (Aurora, OH, USA); NS-398 [N-(2-cyclohexyloxy-4nitrophenyl)-methane sulphonamide], Curcumin, Ibuprofen, Sulindac, 3-(4,5dimethylthiazol-2-yl)-2,5-diphenyltetrazolium bromide (MTT) and Verapamil were purchased from Sigma, St. Louis, MO, USA; metaphosphoric acid was from Aldrich Chemical Co. (Milwaukee, WI, USA). All chemicals and drugs were freshly dissolved before use, except for doxo, which was stored in a stock solution of $1 \mathrm{mM}$ at $4^{\circ} \mathrm{C}$ in the dark.

Cell line and culture conditions. The doxo-resistant human uterus sarcoma cell line (MES-SA/Dx-5) was obtained from European Collection of Cell Culture (Salisbury, UK) and the cells were grown in $25 \mathrm{~cm}^{2}$ culture flask (Iwaki Company, Japan) containing $15 \mathrm{ml}$ McCoy's 5a medium (Euroclone, Wetherby, West Yorkshire, UK) supplemented with 10\% heat-inactivated FBS (Euroclone), $2 \mathrm{mM}$ L-glutamine in a humidified atmosphere of $95 \%$ air and $5 \%$ and $\mathrm{CO}_{2}$ at $37^{\circ} \mathrm{C}$. Cells were sub-cultured every 3-4 days by treatment with $0.05 \%$ trypsin and $0.02 \%$ EDTA in $\mathrm{Ca}^{++}$and $\mathrm{Mg}^{++}$free PBS. Cells were periodically checked for Mycoplasma contamination.

Intracellular accumulation of doxorubicin. For doxo accumulation measurements, attached cells were collected by tripsinization and washed three times with PBS. Aliquots of $1 \mathrm{ml}$ of cells suspension containing $5 \times 10^{5}$ cells were incubated for $24 \mathrm{~h}$ in growth medium in a polystyrene round-bottom tubes (Iwaki Company) at $37^{\circ} \mathrm{C}$ in $5 \% \mathrm{CO}_{2}$. After this period, the cells were pre-treated with $\operatorname{Ver}(10 \mu \mathrm{M})$ or NSAIDs $(10 \mu \mathrm{M})$ for $24 \mathrm{~h}$ at $37^{\circ} \mathrm{C}$ without replacing the media. The cells were then treated with two different concentrations of doxo $(5$ and $10 \mu \mathrm{M})$ for $3 \mathrm{~h}$ at $37^{\circ} \mathrm{C}$ with frequent shaking. Each measurement was performed in triplicate. After incubation, cells were washed twice in icecold PBS (pH 7.4) and after sonication the cell lysates were stored in liquid nitrogen until analysis. After thawing, $3 \mathrm{ml}$ butanol were added and allowed to extract the doxo for $2 \mathrm{~h}$ at $37^{\circ} \mathrm{C}$. After extraction, $1 \mathrm{ml}$ water was added and the phases were mixed vigorously on a vortex mixer and separed by centrifugation. The fluorescence of doxo in the lysates was determined in the butanol phase by fluorescence spectrometry (SFM 25, Kontron Inst.) using 475 and $580 \mathrm{~nm}$ as excitation and emission wavelength, respectively. Extracts from untreated cells were used as blank. Accumulate doxo in the cancer cells pre-treated with either NSAIDs or Ver, was expressed as a percentage of the doxo remaining in control cells (doxo alone) at the selected concentration and measured using the following formula: Doxo accumulation $(\%)=100 \times(\mathrm{F}$ treated $-\mathrm{F}$ control $) / \mathrm{F}$ treated.
Where F treated, mean fluorescence of tubes treated with NSAIDs or Ver prior doxo treatment; F control, mean fluorescence of tubes treated with doxo alone.

Cell toxicity assay. The cytotoxicity of the NSAIDs singly or in combination with doxo, was assessed using MTT assay (29). Briefly, MES-SA/Dx-5 cells were seeded into 96-well culture plates (Iwaki Company) at a density of $1 \times 10^{4}$ cells/ well in growth medium and incubated at $37^{\circ} \mathrm{C}$ for $24 \mathrm{~h}$. Thereafter, the cells were incubated with doxo at various concentrations (5 and $10 \mu \mathrm{M})$ in the absence or presence of NSAIDs $(10 \mu \mathrm{M})$ or Ver $(10 \mu \mathrm{M})$ and incubated at $37^{\circ} \mathrm{C}$ for $72 \mathrm{~h}$. Untreated MES-SA/Dx-5 cells containing medium alone were used as control. The cytoxicity of each drug itself was also measured. After incubation, cells were washed with PBS before $100 \mu 1$ of MTT $(0.5 \mathrm{mg} / \mathrm{ml})$ in drug-free medium was added to each culture well and they were then incubated for an additional $4 \mathrm{~h}$ at $37^{\circ} \mathrm{C}$. The tetrazolium salt MTT is reduced metabolically by living cells to a colored formazan product, which can be quantified spectrophotometrically. Cell media were removed and formazan crystals were solubilized in $100 \mu 1$ of $0.04 \mathrm{~N} \mathrm{HCl}-$ isopropyl alcohol before absorbance was read at $550 \mathrm{~nm}$ on a microplate spectrophotometer (Molecular Devices, Spectra Max 190). Each drug concentration was tested in triplicate. The percentage of growth inhibition was calculated as: Growth inhibition $(\%)=(1-\mathrm{OD}$ treated well/OD control well $)$ $\mathrm{x} 100$. Where OD treated well were the mean absorbance values of cells treated with NSAIDs or Ver while OD control well were the mean values of the untreated cells, respectively.

Percent survival, at each doxo concentration, was expressed as the percent of the control wells and calculated as follows: Cell survival $(\%)=100$ (OD treated well/OD control well.

Apoptosis assays. In order to evaluate whether the combinations of doxo with NSAIDs induced apoptosis, MES-SA/Dx-5 cells were seeded on glass coverslips in 24-well plates at a cell density of $5 \times 10^{4}$ cells $/ \mathrm{ml}$ per well and incubated for 4 days at $37^{\circ} \mathrm{C}$ in a humidified $5 \% \mathrm{CO}_{2}$ atmosphere. Attached cells were then treated for $24 \mathrm{~h}$ with NSAIDs $(10 \mu \mathrm{M})$ in combination with $5 \mu \mathrm{M}$ or $10 \mu \mathrm{M}$ doxo concentrations. Cells grown in drug-free medium were used as control. For assessment of apoptosis we used the acridine orange-propidium iodide dual staining of living cells. The mixture of fluorescence dyes consisted of acridine orange at $5 \mu \mathrm{g} / \mathrm{ml}$ and propidium iodide at $5 \mu \mathrm{g} / \mathrm{ml}$ in PBS. Cells were stained for $10 \mathrm{~min}$ at RT, washed twice with PBS and immediately viewed under a confocal microscope (Zeiss LSM 510 META). In early apoptosis, acridine orange enters the cells and nuclei are bright with dense green areas. Propidium iodide only stains DNA red of cells in late stages of apoptosis and necrotic cells with alterated membrane integrity. Each treatment was repeated in triplicate.

Statistical analysis. The presented data were expressed as a mean \pm SD and compared with the Student t-test. P-values at $<0.05$ were considered statistically significant. 


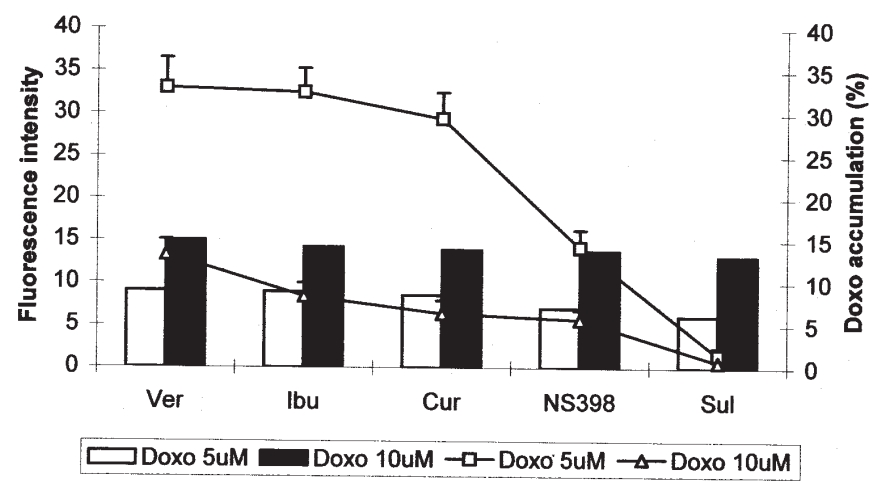

Figure 1. Mean retained intracellular doxo fluorescence intensities in resistant MES-SA/Dx-5 cells pretreated with the NSAIDs or Ver after incubation with two different doxo concentrations. The mean percentage of increase of cellular doxo accumulation in NSAIDs and Ver-treated cells compared to the untreated cells, is also reported. Each point represents the mean of triplicate determinations.

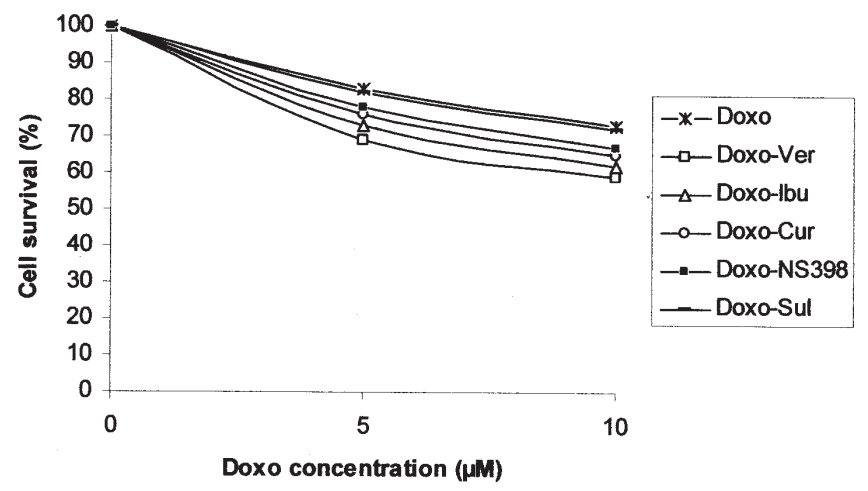

Figure 2. Mean percentage of surviving cells determined in MES-SA/Dx-5 cells after treatment with doxo alone (asterisk) and in combination with either NSAIDs or Ver at the indicated concentrations. The results are corrected for the toxicity of each drug in resistant cancer cells (Materials and methods). Data are the mean of three determinations.

\section{Results}

Accumulation assay. Fig. 1 shows the increase of intercellular doxo accumulation in cancer cells pre-treated for $24 \mathrm{~h}$ at $37^{\circ} \mathrm{C}$ with NSAIDs or Ver compared to those cultures not previously exposed to inhibitors. As shown, the co-administration of NSAIDs enhanced the doxo accumulation capacity in MESSA/Dx-5 cells at all doxo concentrations. In particular, the average percentage of doxo retention after exposure at 5 and $10 \mu \mathrm{M}$ doxo concentrations in the presence of Ibu, Cur, NS-398 and Sul reached 32.1 $\pm 2.8,29.4 \pm 3.1,14.2 \pm 2.1$, $1,5 \pm 0.9 \%$ and $8.4 \pm 1.7,6.4 \pm 1.5,5.7 \pm 1.2,0.7 \pm 0.5 \%$, respectively, when compared to control cells, receiving only medium. As reported in Fig. 1, a significant increase in the accumulation of doxo $(\mathrm{p}<0.05)$ was observed in cells pretreated with Ibu and Cur that were able to retain intracellular doxo level superior to $30 \%$ of the control after exposure to $5 \mu \mathrm{M}$ doxo concentration. These results were similar to that obtained when the cells were incubated with conventional sensitizer, Ver. At the same doxo concentration the mean doxo accumulation for NS-treated cells was $\sim 15 \%$ of control,
Table I. Cytotoxic effects of doxo alone and combined with the NSAIDs or Ver in MES-SA/Dx-5 cells.

\begin{tabular}{lcc}
\hline Treatment & $\mathrm{A}_{550 \mathrm{~nm}}$ & $\begin{array}{c}\text { Growth } \\
\text { inhibition }(\%)\end{array}$ \\
\hline Control & 1.26 & - \\
Doxo $5 \mu \mathrm{M}$ & $1.05 \pm 0.04$ & $17 \pm 2.3$ \\
Doxo $10 \mu \mathrm{M}$ & $0.93 \pm 0.03$ & $27 \pm 2.5$ \\
Ver $10 \mu \mathrm{M}$ & $1.22 \pm 0.03$ & $4 \pm 0.9$ \\
Ibu $10 \mu \mathrm{M}$ & $1.24 \pm 0.02$ & $2 \pm 0.8$ \\
Cur $10 \mu \mathrm{M}$ & $1.25 \pm 0.02$ & $1 \pm 0.5$ \\
NS $10 \mu \mathrm{M}$ & $1.25 \pm 0.02$ & $1 \pm 0.5$ \\
Sul $10 \mu \mathrm{M}$ & $1.25 \pm 0.01$ & $1 \pm 0.4$ \\
Doxo $5 \mu \mathrm{M}+\operatorname{Ver} 10 \mu \mathrm{M}$ & $0.82 \pm 0.03$ & $35 \pm 2.5$ \\
Doxo $5 \mu \mathrm{M}+$ Ibu $10 \mu \mathrm{M}$ & $0.90 \pm 0.03$ & $29 \pm 2.6$ \\
Doxo $5 \mu \mathrm{M}+\operatorname{Cur} 10 \mu \mathrm{M}$ & $0.95 \pm 0.02$ & $25 \pm 1.8$ \\
Doxo $5 \mu \mathrm{M}+\mathrm{NS} 10 \mu \mathrm{M}$ & $0.98 \pm 0.03$ & $23 \pm 2.1$ \\
Doxo $5 \mu \mathrm{M}+\operatorname{Sul} 10 \mu \mathrm{M}$ & $1.04 \pm 0.05$ & $17 \pm 3.2$ \\
Doxo $10 \mu \mathrm{M}+\operatorname{Ver} 10 \mu \mathrm{M}$ & $0.70 \pm 0.02$ & $45 \pm 2.5$ \\
Doxo $10 \mu \mathrm{M}+\operatorname{Ibu} 10 \mu \mathrm{M}$ & $0.76 \pm 0.03$ & $40 \pm 2.2$ \\
Doxo $10 \mu \mathrm{M}+\operatorname{Cur} 10 \mu \mathrm{M}$ & $0.81 \pm 0.04$ & $36 \pm 1.4$ \\
Doxo $10 \mu \mathrm{M}+\mathrm{NS} 10 \mu \mathrm{M}$ & $0.84 \pm 0.03$ & $34 \pm 2.8$ \\
Doxo $10 \mu \mathrm{M}+\operatorname{Sul} 10 \mu \mathrm{M}$ & $0.91 \pm 0.02$ & $27 \pm 1.5$ \\
\hline
\end{tabular}

while the accumulated amount of doxo in resistant cells changed little in the presence of sulindac (only 1-2\%). At $10 \mu \mathrm{M}$, intracellular doxo concentration dropped to values $<10 \%$ for NSAIDs and only Ver was still able to maintain intracellular doxo levels superior to $10 \%$ to control cells.

Cytotoxicity assay. In Table I are reported the values of cytotoxic activity of doxo on cancer cells at different concentrations when incubated in the absence or presence of NSAIDs or Ver for $72 \mathrm{~h}$ at $37^{\circ} \mathrm{C}$. As shown in Table I, the growth inhibition using doxo 5 and $10 \mu \mathrm{M}$ concentrations in combination with Ibu, Cur, NS-398 and Sul resulted in 1.7-, 1.4-, 1.3- and 1.1-fold and 1.5-, 1.3-, 1.2- and 1.1-fold increase, respectively. These results demonstrated that the combinations of doxo with NSAIDs enhance the sensitivity of cancer cells to doxo treatment when compared to cells treated with doxo alone revealing a synergistic effect in this resistant cancer cells. In addition, by themselves NSAIDs did not show relevant toxic effects on MES-SA/Dx-5 cells while the toxicity of Ver alone was $\sim 3$-fold higher than those observed in the NSAID-treated cells. The percentage of cell survival calculated by subtracting the corresponding cytotoxicity values of the agents alone from the respective values achieved in association with all doxo concentrations is shown in Fig. 2.

Apoptosis assay. All combinations examined enhanced apoptosis characterized by cell shrinkage and nuclear condensation when compared to control cells (without inhibitors). The highest number of early apoptotic cells was observed in doxo-Ver combinations (Fig. 3b). However, the 

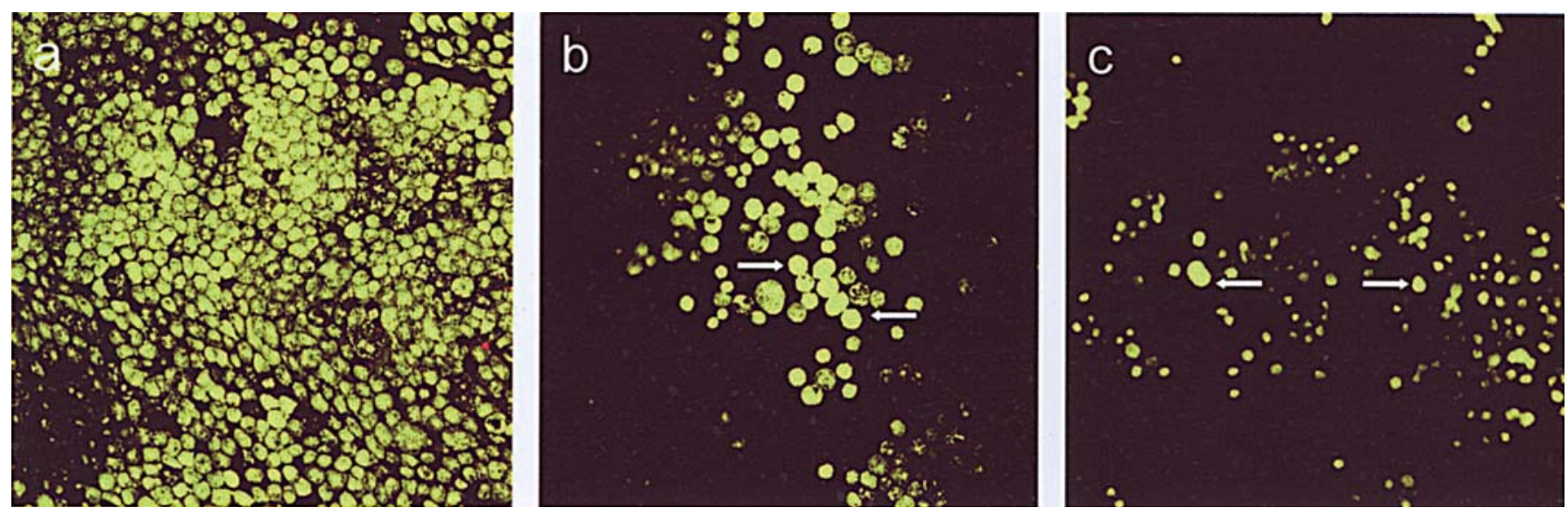

Figure 3. Representative view of acridine orange-propidium iodide staining of living MES-SA/Dx-5 cells (x20 magnification) before (a) and following exposure to $5 \mu \mathrm{M}$ doxo combined with $10 \mu \mathrm{M}$ Ver (b) or $10 \mu \mathrm{M}$ Ibu (c) for $24 \mathrm{~h}$ as described in Materials and Methods. Arrows indicate early apoptotic cells.

induction of apoptosis was significantly enhanced in cells treated with Ibu in combination with doxo (Fig. 3c) and gradually decreased in Cur and NS-treated cells. While little change was observed in MES-SA/Dx-5 cells after exposure to Sul-doxo combinations, compared to the control. These results suggest that the enhancement of cytotoxic activity of doxo could be related to a concomitant increase of apoptosis.

\section{Discussion}

P-gp overexpression confers resistance to a wide variety of anti-cancer agents used for treatment of different types of neoplastic diseases. At present, none of the P-gp inhibitors tested in vitro to block P-gp activity has been introduced in the clinical oncological practice. In this study, we have demonstrated that a group of clinically important NSAIDs at concentrations achievable in human serum, significantly enhance the sensitivity to doxo in human sarcoma MES-SA/ Dx-5 cells which overexpress P-gp, when compared to untreated cells (without inhibitors). As shown in Fig. 1, all four anti-inflammatory agents were able to increase the intracellular accumulation of doxo compared to cells treated with doxo alone. In particular, the cells pre-treated with $5 \mu \mathrm{M}$ doxo in combination with Ibu or Cur have shown a significant increase in intracellular doxo accumulation (up to 30\%) compared to the control $(\mathrm{p}<0.05)$. A significant increase (15\%) was also obtained after pre-treatment with NS-398, while the lowest increase of doxo retention (1-2\%) was achieved in Sul-treated cells. At higher doxo concentrations $(10 \mu \mathrm{M})$, surprisingly, the capacity to retain doxo dropped $<10 \%$ for all NSAIDs tested, remaining $~ 15 \%$ only for conventional P-gp inhibitor, Ver. These results suggest that a relationship between NSAIDs and the P-gp activity in the resistant sarcoma cells exists and that the increase of cellular doxo retention appears to be independent of the extracellular doxo concentration. However, the exact molecular mechanism(s) to explain this phenomenon remain to be established. A possible explanation is that these lipophilic molecules accumulate within the cancer cells and at low concentrations of doxo, act as P-gp substrates competing actively with doxo for binding sites on $\mathrm{P}-\mathrm{gp}$ and thus increasing its intracellular accumulation. In contrast, at high doxo concentrations, MES-SA/Dx-5 cells extrude mainly doxo reducing the interaction with the NSAIDs and consequently decreasing intracellular doxo levels. This hypothesis agrees with the results obtained with MTT test. Indeed, as shown in Table I all four anti-inflammatory agents enhanced the cytotoxic effects of doxo on cancer cells more effectively in the presence of low doxo concentrations, when compared to the controls. In addition, these results are consistent with the increased apoptotic response obtained when MES-SA/Dx-5 cells were treated concomitantly with doxo and NSAIDs, indicating that the further reduction of cell proliferation could be caused in part, by an apoptotic mechanism. In summary, our results demonstrate that Ibu, Cur and NS greatly increase the sensitivity to doxo in MES-SA/ Dx-5 cells via modulation with P-gp activity at concentrations $(2.9 \mu \mathrm{g} / \mathrm{ml})$ that are much lower than those of plasma attainable levels when standard doses of doxo are administered clinically $(8-10 \mu \mathrm{g} / \mathrm{ml})$. These findings have a clinical importance because through a combined chemotherapy of doxo and NSAIDs reduced doxo concentration and its side effects (cardiotoxicity, myelosuppression, and other systemic side effects) could be possible without reducing its cytotoxic activity (30). Furthermore, since these three compounds exert their sensitizing effects in vitro well below their therapeutic plasma concentrations $(2-3 \mu \mathrm{g} / \mathrm{ml})$, their co-administration at very low dosages (side effect free) could enhance the efficacy of doxo-based regimens in the treatment of P-gp-mediated MDR tumors. In contrast, Sul was the weakest inhibitor of Pgp activity and only slightly effective as chemosensitizer, since the concentration necessary to inhibit effectiveness in vitro growth (>100 $\mu \mathrm{M}$ data not shown) of MES-SA/Dx-5resistant cells, was higher than serum concentrations attainable in vivo $(\sim 10 \mu \mathrm{M})$ at clinical dosages. In conclusion, we found that Ibu, Cur and NS are effective chemosensitizers with activity similar to that of the known P-gp-modulator Ver but with much less in vivo toxic effects (arrhythmia and hypotension). Furthermore, the discovery and the development of new NSAIDs or their derivatives with minimal toxicity and without side effects (platelet function or gastrointestinal effect) could increase the clinical use of this class of drugs as 
a new potential generation of chemosensitizing agents to prevent or reverse drug resistance in human cancer.

\section{Acknowledgements}

We are grateful to Massimo Ruzzi and Christian Mazzocco for their technical assistance.

\section{References}

1. Ling V: Multidrug resistance: molecular mechanism and clinical relevance. Cancer Chemother Pharmacol 40: 3-8, 1997.

2. Juliano RL and Ling V: A surface glycoprotein modulating drug permeability in Chinese hamster ovary cell mutants. Biochim Biophys Acta 455: 152-162, 1976.

3. Pastan I and Gottesman MM: Multiple-drug resistance in human cancer. N Engl J Med 316: 1388-1393, 1987.

4. Ambudkar SV, Dey S, Hrycyna CA, Ramachandra M, Pastan I and Gottesman MM: Biochemical, cellular, and pharmacological aspects of the multidrug transporter. Annu Rev Pharmacol Toxicol 39: 361-398, 1991 .

5. Nooter K and Stoter G: Molecular mechanism of multidrug resistance in cancer chemotherapy. Pathol Res Pract 192: 768-780, 1996.

6. Gottesman MM, Fojo T and Bates SE: Multidrug resistance in cancer: role of ATP-dependent transporters. Nat Rev Cancer 2: 48-58, 2002.

7. Seeber S, Asieka R, Schmidt CG, Achterrath W and Crooke GT: In vivo resistance towards anthracycline, etoposide and cisdiaminedichloroplatinum. Cancer Res 42: 4719-4725, 1982.

8. Endicott JA and Ling VZ: The biochemistry of P-glycoprotein mediated multidrug resistance. Annu Rev Biochem 58: 137-171, 1989.

9. Higgins $\mathrm{CF}$ : $\mathrm{ABC}$ transporters: from microorganisms to man. Annu Rev Cell Biol 8: 67-113, 1992.

10. Raviv Y, Pollard HB, Bruggermann EP, Pastan I and Gottesman MM: Photosensitized labeling of a functional multidrug transporter in living drug-resistant tumor cells. J Biol Chem 265: 3975-3980, 1990.

11. Sauna ZE, Smith MM, Muller M, Kerr KM and Ambudkar SV: The mechanism of action of multidrug-resistance-linked Pglycoprotein. J Bioenerg Biomembr 33: 481-491, 2001.

12. Krishna R and Mayer LD: Multidrug resistance (MDR) in cancer. Mechanisms, reversal using modulators of MDR and role of MDR modulators in influencing the pharmacokinetics of anticancer drugs. Eur J Pharm Sci 11: 265-283, 2000.

13. Borowski E, Bontemps-Gracz MM and Piwkowska A: Strategies for overcoming ABC-transporters-mediated multidrug resistance (MDR) of tumor cells. Acta Biochim Pol 52: 609-627, 2005.

14. Thomas H and Coley HM: Overcoming multidrug resistance in cancer: An update on the clinical strategy of inhibiting Pglycoprotein. Cancer Control 10: 159-165, 2003.
15. Sarkadi B and Müller M: Search for specific inhibitors of multidrug resistance in cancer. Semin Cancer Biol 8: 171-182, 1997.

16. Twentymann PR and Bleehen NM: Resistance modification by PSC 833, a novel non-immunosuppressive cyclosporine. Eur J Cancer 7: 1639-1642, 1991.

17. Bardelmeijer HA, Beijnen JH, Brouwer KR, Rosing H, Nooijen WJ, Shellens JH and van Tellingen O: Increased oral bioavailability of paclitaxel by GF120918 in mice through selective modulation of P-glycoprotein. Clin Cancer Res 6: 4416-4421, 2000.

18. Green LJ, Marder P and Slapak CA: Modulation by LY335979 of P-glycoprotein function in multidrug-resistant cell lines and human natural killer cells. Biochem Pharmacol 61: 1393-1399, 2001.

19. Sikic BI, Fisher GA, Lum BL, Halsey J, Beketic-Oreskovic L and Chen G: Modulation and prevention of multidrug resistance by inhibitors of P-glycoprotein. Cancer Chemother Pharmacol 40: 13-19, 1997

20. Andrews P, Zhao X, Allen J, Fengmin L and Chang M: A comparison of the effectiveness of selected non-steroidal antiinflammatory drugs and their derivatives against cance cells in vitro. Cancer Chemother Pharmacol 61: 203-214, 2008.

21. Zha S, Yegnasubramanian V, Nelson WG, Isaac WB and De Marzo AM: Cyclooxygenase in cancer: progress and perrpective. Cancer Lett 215: 1-20, 2004.

22. Meric JB, Rottey S, Olaussen K, Soria JC, Khayat D, Rixe O and Spano JP: Cyclooxygenase-2 as a target for anticancer drug development. Crit Rev Oncol Hematol 59: 51-64, 2006.

23. Smith ML, Hawcroft G and Hull MA: The effect of non-steroidal anti-inflammatory drugs on human colorectal cancer cells: evidence of different mechanisms of action. Eur J Cancer 36: 664-674, 2000

24. Howe LR and Dannenberg AJ: A role for cyclooxygenase-2inhibitors in the prevention and treatment of cancer. Semin Oncol 29: 111-119, 2002.

25. Guadagni F, Ferroni P, Palmirotta R, Del Monte G, Formica V and Roselli M: Non-steroidal anti-inflammatory drugs in cancer prevention and therapy. Anticancer Res 27: 3147-3162, 2007.

26. Harker WG and Sikic BI: Multidrug (pleiotropic) resistance in doxorubicin-selected variants of the human sarcoma cell line MES-SA. Cancer Res 45: 4091-4096, 1985.

27. Wesolowska O, Paprocka M, Koziak J, Motohashi N, Dus D and Michalak K: Human sarcoma cell lines MES-SA and MESSA/Dx5 as a model for multidrug resistance modulators screening. Anticancer Res 25: 383-389, 2005.

28. Tsuruo T, Iida H, Tsukagoshi S and Sakurai Y: Overcoming of vincristine resistance in P388 leukemia in vivo and in vitro through enhanced cytotoxicity of vincristine and vinblastine by verapamil. Cancer Res 41: 1967-1972, 1981.

29. Mosmann T: Rapid colorimetric assay for cellular growth and survival: application to proliferation and cytotoxicity assay. J Immunol Methods 65: 55-63, 1983.

30. Shan K, Lincoff AM and Young JB: Anthracycline-induced cardiotoxicity. Ann Intern Med 125: 47-58, 1996. 